\title{
Rippled $\beta$-Sheet Fibrils from Coassembled Enantiomeric Amphipathic Peptides as Potential Microbicide Biomaterials
}

\author{
Bradley L. Nilsson, Danielle M. Raymond, and Jade J. Welch \\ University of Rochester, Department of Chemistry, Rochester, NY, 14618, USA
}

\section{Introduction}

Amphipathic $\beta$-sheet peptides that assemble into cross- $\beta$ bilayer fibrils have been widely applied as functional biomaterials [1]. We recently discovered that enantiomeric D- and L-Ac-(FKFE) ${ }_{2}-\mathrm{NH}_{2}$ peptides readily coassemble into two-component "rippled $\beta$-sheet" fibrils (see Figure 1) [2] (as opposed to classical pleated $\beta$-sheet materials), consistent with Pauling's prediction for mixtures of enantiomeric $\beta$-sheet peptides [3]. Coassembly was confirmed by isotope-edited IR spectroscopy and FRET-based fluorescence spectroscopy. The preference for coassembly into rippled $\beta$-sheets relative to self-sorting into pleated $\beta$-sheets is thermodynamically favored. Rippled $\beta$-sheet fibrils have unique emergent properties compared to pleated $\beta$-sheet fibrils, including enhanced hydrogel elasticity and resistance to protease degradation [4].

A

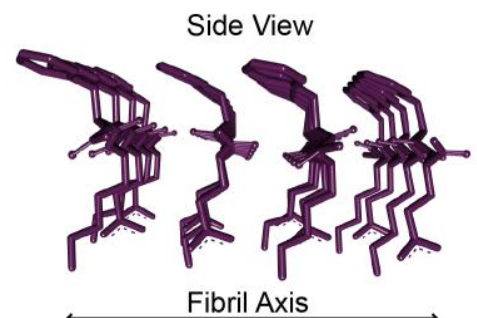

B



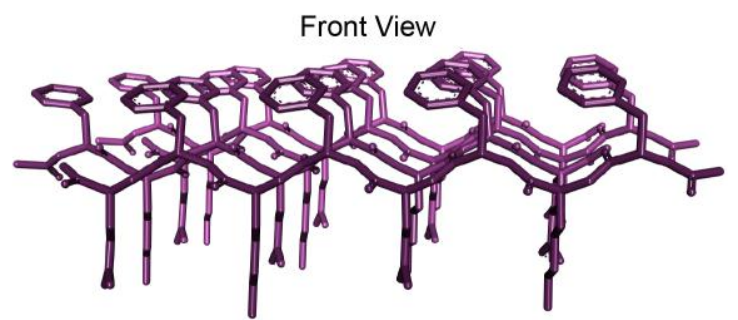

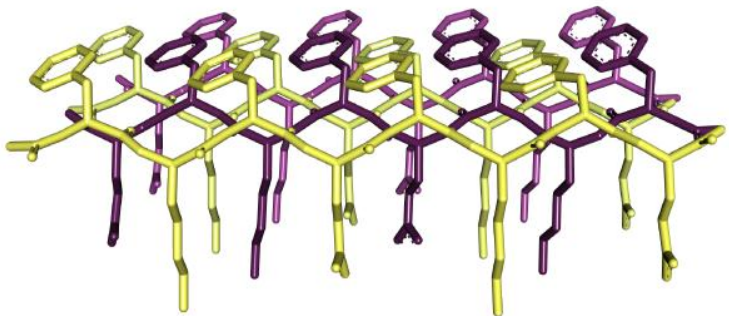

Fig. 1. A. Representative self-assembled pleated $\beta$-sheets of $L-A c-(F K F E)_{2}-N_{2} H_{2}$. The fibril axis is parallel to the plane of the page as shown in the left image; the fibril axis is perpendicular to the page in the image to the right. Fibrils of this peptide are composed of the bilayer of the indicated $\beta$-sheets. $B$. Representative pleated $\beta$-sheets of coassembled $L$ - and D-Ac- $(F K F E)_{2}-N_{2} H_{2}$ peptides. The L-peptide is shown in purple and the D-peptide is shown in yellow.

Engineered rippled $\beta$-sheet scaffolds have potential for application as multicomponent noncovalent materials. There is a need for novel hydrogel materials that can be exploited as microbicides to prevent the sexual transmission of HIV. Recent studies indicate that hydrogels commonly used as personal lubricants are irritating to vaginal and rectal mucosa leading to fears that these materials may enhance the spread of HIV and other sexually transmitted diseases [5]. Ideal hydrogel materials for intravaginal or intrarectal application should be non-irritating and non-immunogenic. Nanofibrils of Ac-(FKFE $)_{2}-\mathrm{NH}_{2}$ and related amphipathic peptides have been shown to be non-immunogenic, supporting their potential for application as vaginal or rectal microbicides [6]. In addition, ideal microbicides should be deliverable, shear-responsive hydrogels that maintain integrity in vaginal and rectal environments. These materials must also facilitate the multivalent display of functional groups that are accessible to interact with HIV virions or target cells. Finally, microbicides must exhibit stability to proteolytic 
degradation. Herein, we discuss the properties of rippled $\beta$-sheet derived from amphipathic enantiomeric peptides that suggest the potential of these as hydrogel materials targeted to the prevention of sexually transmitted diseases, including HIV.

\section{Results and Discussion}

We recently reported that enantiomeric amphipathic peptides undergo coassembly to form rippled $\beta$-sheet structures [2]. Specifically, we discovered that equimolar mixtures of D- and L-Ac-(FKFE) ${ }_{2}-\mathrm{NH}_{2}$ peptides selectively coassemble into two-component rippled $\beta$-sheets as opposed to assembling into self-sorted, single enantiomer pleated $\beta$-sheet fibrils. Coassembly was confirmed by isotope-edited IR spectroscopy and FRET analysis in which the enantiomers were labeled with acceptor and donor fluorophores. The mechanistic basis for preferential coassembly of enantiomers was also explored. We conducted isothermal titration calorimetry experiments using the L-Ac- $(\mathrm{FEFE})_{2}-\mathrm{NH}_{2}$ peptide and Land D-Ac-(FKFK $)_{2}-\mathrm{NH}_{2}$ peptides. These cationic and anionic peptides fail to self-assemble due to charge repulsion at neutral $\mathrm{pH}$. However, when peptides of opposite charge are mixed they readily coassemble. Either L- or D-Ac-(FKFK) $)_{2}-\mathrm{NH}_{2}$ peptides were titrated into L-Ac-(FEFE) $)_{2}-\mathrm{NH}_{2}$. The L/L mixture provided pleated $\beta$-sheets while the L/D mixture provided rippled $\beta$-sheets. It was found that rippled $\beta$-sheet formation had an $\sim 9 \mathrm{kcal} \mathrm{mol}^{-1}$ enthalpic advantage over pleated $\beta$-sheet formation.

The structural basis for this energetic advantage for rippled $\beta$-sheet formation is not completely understood. One possible explanation lies in differences for $\beta$-sheet strand registry between pleated and rippled sheets for the Ac-(FKFE) $)_{2}-\mathrm{NH}_{2}$ peptide. For pleated $\beta$-sheet formation from self-assembly of single enantiomers it has been proposed that the strand alignment requires the $N$-terminal Phe residue to be out of register [7], leaving hydrogen bond donors and acceptors unsatisfied as well as less than optimal pairing of the dangling hydrophobic side chain. In rippled $\beta$-sheets formed from enantiomeric Ac-(FKFE) $)_{2}-\mathrm{NH}_{2}$ peptides, the putative $\beta$-sheet alignment is completely in register, resulting in structures with additional stabilizing hydrogen bonds. In addition, the side chain arrangement of rippled $\beta$-sheets differs from that observed in pleated $\beta$-sheets. In pleated sheets, crossstrand side chain groups exist in an eclipsed-like conformation (Figure 1A) whereas rippled sheets orient the side chain groups in a staggered-like conformation. These differences in cross-strand side chain orientation may also contribute to the significant enthalpic advantage for rippled $\beta$-sheets. Additional studies are ongoing to elucidate the precise physicochemical basis for preferential rippled $\beta$-sheet formation.

We have also found that rippled $\beta$-sheets derived from enantiomeric Ac-(FKFE) $)_{2}-\mathrm{NH}_{2}$ peptides have protease degradation profiles that make them attractive candidates for microbicide hydrogels. Specifically, we have reported that rippled $\beta$-sheets resist degradation from common proteases, including trypsin, chymotrypsin, and proteinase $\mathrm{K}$ [4]. We formed hydrogels from Land D-Ac-(FKFE) $)_{2}-\mathrm{NH}_{2}$ peptides: pleated $\beta$-sheet hydrogels from either $\mathrm{L}-$ or D-Ac-(FKFE) $)_{2}-\mathrm{NH}_{2}$ and rippled $\beta$-sheet hydrogels from coassembled Land $\mathrm{D}-\mathrm{Ac}-(\mathrm{FKFE})_{2}-\mathrm{NH}_{2}$. It was found that pleated $\beta$-sheet hydrogels derived from L-Ac-(FKFE) $)_{2}-\mathrm{NH}_{2}$ were completely degraded by these proteases in hours. In contrast, the rippled $\beta$-sheet hydrogels were as resistant to the proteases as the all-D pleated $\beta$-sheet fibrils (Figure 2).

Thus, rippled $\beta$-sheet hydrogels are exceptionally stable materials that meet several of the criteria necessary for the creation of novel anti-HIV microbicides that prevent sexual transmission of the virus. We have

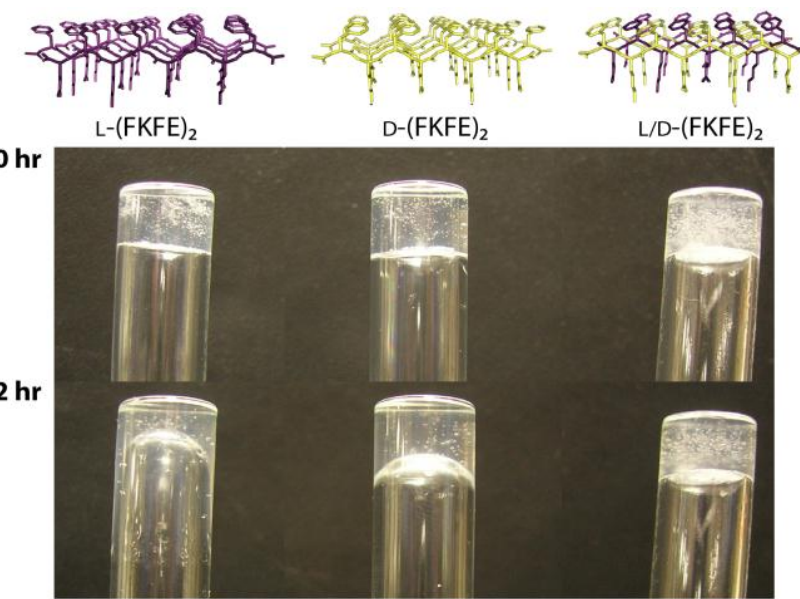

Fig. 2. Digital images of hydrogels incubated with chymotrypsin showing degradation of the L-pleated sheet material after several hours. Rippled $\beta$-sheet hydrogels were stable to degradation even after 5 days. 
previously shown that pleated $\beta$-sheets that display the entry inhibitor aplaviroc also have specific antiHIV properties [8]. Appropriate modification of rippled $\beta$-sheet materials should also result in similar properties that elicit specific protection against HIV. Work is ongoing to establish the immunogenicity of rippled $\beta$-sheet materials. While additional development is necessary, rippled $\beta$-sheets have great potential as novel, next-generation hydrogel biomaterials for the prevention of sexually transmitted diseases that avoid the problems with existing commercial hydrogels.

\section{Acknowledgments}

The National Science Foundation (DMR-1148836) supported this work.

\section{References}

1. Bowerman, C.J., Nilsson, B.L. Biopolymers 98, 169-184 (2012), http://dx.doi.org/10.1002/bip.22058

2. Swanekamp, R.J., DiMaio, J.T.M., Bowerman, C.J., Nilsson, B.L. J. Am. Chem. Soc. 134, 5556-5559 (2012), http://dx.doi.org/10.1021/ja301642c

3. Pauling, L., Corey, R.B. Proc. Natl. Acad. Sci. USA 39, 253-256 (1953).

4. Swanekamp, R.J., Welch, J.J., Nilsson, B.L. Chem. Commun. 50, 10133-10136 (2014),

http://dx.doi.org/10.1039/C4CC04644G

5. Wolf, L.K. Chem. \& Eng. News 90, 46-47 (2012).

6. Rudra, J.S., Sun, T., Bird, K.C., Daniels, M.D., Gasiorowski, J.Z., Chong, A.S., Collier, J.S. ACS Nano 6, 1557-1564 (2012), http://dx.doi.org/10.1021/nn204530r

7. Hwang, W., Marini, D.M., Kamm, R.D., Zhang, S. J. Chem. Phys. 118, 389-397 (2003), http://dx.doi.org/10.1063/1.1524618

8. DiMaio, J.T.M., Easterhoff, D., Moore, A.M., Dewhurst, S., Nilsson, B.L., in Lebl, M. (Ed.) Peptides: Peptides Across the Pacific (Proceedings of the 23rd American Peptide Symposium), American Peptide Society, San Diego, 2013, pp. 154-155, http://dx.doi.org/10.17952/23APS.2013.154 